\title{
Distinct brain representations for early and late learned words
}

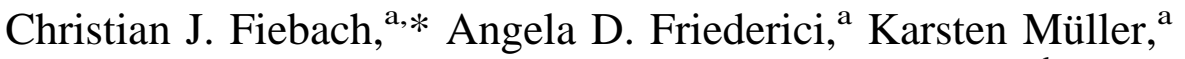 \\ D. Yves von Cramon, ${ }^{a}$ and Arturo E. Hernandez ${ }^{a, b}$ \\ ${ }^{a}$ Max Planck Institute of Cognitive Neuroscience, Leipzig, Germany \\ ${ }^{\mathrm{b}}$ Department of Psychology, University of California, Santa Barbara, CA, USA
}

Received 18 October 2002; revised 12 March 2003; accepted 14 April 2003

\begin{abstract}
Recently there has been a renewed interest in cognitive psychology on the effects of the age of word acquisition (AoA) on lexical processing. In particular, it is currently unclear whether AoA or word frequency are better predictors of word recognition. To date no study has investigated the neural bases of the AoA effect or attempted to dissociate it from word frequency. We report a visual and an auditory event-related fMRI experiment investigating the influence of AoA and word frequency on neural activity, and show that AoA modulates brain areas that are not influenced by word frequency. The precuneus was activated for early learned words across auditory and visual presentation modalities. Additional activity in the auditory cortex was observed specifically for the reading of early acquired words. Late learned words, in contrast, led to a selective activation increase in lateral inferior frontal areas. These findings support models that suggest that early and late learned words are represented differently in the brain. They further allow to specify the nature of the represenational differences, namely that early learned words are represented in the brain in a more sensory manner than late learned words.

(C) 2003 Elsevier Science (USA). All rights reserved.
\end{abstract}

\section{Introduction}

A central issue in cognitive neuroscience centers on understanding how the brain processes words. Research using functional imaging methods such as positron emission tomography (PET) and functional magnetic resonance imaging (fMRI) have yielded considerable insight into the networks of cortical areas involved in the process of word recognition (e.g., Petersen et al., 1988; Norris and Wise, 2000; Price, 2000). Psycholinguistic research has demonstrated that two important independent factors strongly influence how individual words are processed: a word's frequency of occurrence and the age at which a word was learned. Word frequency probably is the most widely investigated variable in word recognition research. Frequently encountered words (such as "weather" or "text") are processed more easily than less common words like "rhyme" or

\footnotetext{
* Corresponding author. Max Planck Institute of Cognitive Neuroscience, Department of Neuropsychology, P.O. Box 500 355, D-04303 Leipzig, Germany. Fax: +49-341-9940-113.

E-mail address: fiebach@cns.mpg.de (C.J. Fiebach).
}

"cue" (e.g., Grainger, 1990; Jescheniak and Levelt, 1994). This finding has been interpreted as reflecting differences in the ease with which cognitive mechanisms can access representations of words in the mental lexicon (e.g., Forster, 1976). Recent neuroimaging studies investigating frequency effects during word processing have helped to more precisely characterize the contribution especially of inferior frontal brain areas to the process of word recognition (Fiez et al., 1999; Fiebach et al., 2002; Chee et al., 2002). The second factor, the age of word acquisition, has long been neglected in the word processing literature. For quite some time, cognitive psychologists have observed that words that were learned early in life, such as "car," are processed differently than later acquired words like, e.g., "mineral" or "text" (Carroll and White, 1973; Gilhooly and Logie, 1982). However, only recently have researchers begun to openly question the relationship between word frequency effects and age of acquisition effects (Morrison and Ellis, 1995; De Moor et al., 2001).

In the last few years, researchers have begun to suggest that age of acquisition of words (AoA) is indeed a more important variable than word frequency, as it allows to 
better predict the individuals' speed and accuracy in a number of psycholinguistic tasks such as word naming and lexical decision (Morrison and Ellis, 1995; De Moor et al., 2001). In light of these findings, different theoretical models have been proposed to explain the effect of AoA on word processing. One suggestion is that words learned early in life are stored with more complete representations in the phonological output lexicon than late acquired words and, therefore, can be articulated faster (phonological completeness hypothesis; Brown and Watson, 1987). Pronouncing late learned words, according to this account, consumes more time as more phonological information has to be generated that is not directly represented in the phonological output lexicon. Other researchers, however, have doubted this approach as it does not capture the whole range of empirical findings (Yamazaki et al., 1997; Lewis et al., 2001). In particular, the phonological completeness hypothesis cannot account for AoA effects in the lexical decision task, in which participants do not have to pronounce the words.

Some researchers have gone so far as to claim that the effects of AoA and word frequency are so confounded that they are virtually impossible to disentangle at the behavioral level. This has led to the suggestion that both effects are the product of similar underlying processes and that previously reported word frequency effects are mostly due to a confound with age of word acquisition (Morrison and Ellis, 1995). Others have taken an opposite theoretical position, claiming that AoA effects merely reflect cumulative frequency of exposure (Lewis et al., 2001).

A recent study using connectionist network modeling of AoA effects (Ellis and Lambon Ralph, 2000) found that AoA effects cannot be explained solely in terms of cumulative frequency. This was concluded because-although both factors had the same outcome on the network structure, i.e., a change in connection strengths - the time at which the network learns a word (i.e., the age at the word's acquisition) appears to affect also the quality of the word's representation, not only the quantitative measures. Early learned words influence the structure of the network in a more profound way. These simulation data show that the disadvantage for later learned words could be due to a gradual loss of plasticity of the cognitive system during development. The simulated neural network is optimized during development for the efficient processing of earlier learned words, and therefore struggled more with later learned words. These results suggest that late learned words will develop mental representations not completely comparable to those of words acquired early in life (Ellis and Lambon Ralph, 2000).

Previous studies in our laboratory have revealed that words of low and high frequency elicit different patterns of neural activity during visual word processing (Fiebach et al., 2002). By using event-related fMRI, we demonstrated that low frequency words evoked greater activity than high frequency words in the inferior frontal lobe of the left

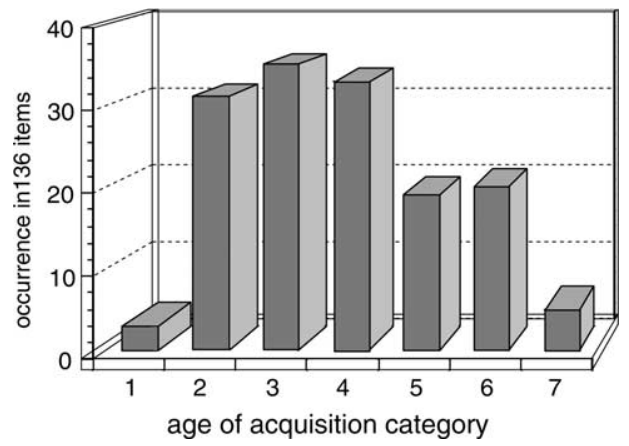

Fig 1. Distribution of age of acquisition (AoA) ratings. Mean age of acquisition ratings $(n=29)$ for the 136 words used in the present study. For display purposes, AoA values were aggregated into the seven categories of acquisition age used in the questionnaire study (see Methods section).

hemisphere, namely in Brodmann areas (BA) 44 and 45, as well as in the anterior insula and in subcortical structures bilaterally (see Chee et al., 2002, for independent evidence supporting the finding of frequency effects in the left inferior frontal cortex). As noted earlier, the discussion in the psychological literature has centered on whether word frequency and age of acquisition effects are the product of distinct processes. In the current study, we investigate whether early and late learned words are represented differently in the brain and, if so, whether brain activation effects for word frequency and AoA are mediated by common neural systems or not. This was done by determining age of acquisition estimates for the word stimuli used in the abovedescribed study (Fiebach et al., 2002) on the basis of internal norms (see Methods section) and then analyzing the fMRI data set in which the above-described frequency effect was obtained in a common statistical model containing both factors, AoA and frequency, as continuous variables.

AoA ratings revealed that the greatest part of the word stimuli in the present set were estimated to be acquired between the ages of 3 and 12 years. In contrast, only very few words were rated as being learned at age 2 or earlier, or after age 13 (Fig. 1). There was no substantial relation between age of acquisition and word length, neither when considering the number of letters $(r=0.15$, NS) nor when correlating AoA with the number of syllables in a word ( $r=$ 0.16 , NS). There was a relatively high negative correlation between AoA and word frequency $(r=-0.58 ; P<0.000)$. Later learned words were on average less common than earlier learned words. To be able to isolate the specific effects of AoA and word frequency on brain activation, independent of the shared variance component, a multiple regression approach was used. This statistical procedure is analogous to the one frequently used in behavioral studies comparing the two factors of interest here (e.g., Morrison and Ellis, 1995; Brown and Watson, 1987). The main goal was to determine the amount of variance in lexical decision performance and functional imaging data accounted for by word frequency and AoA when the respective other factor 
was kept constant. To accomplish this, age of acquisition ratings and word frequency (logarithmic frequency as given in the CELEX database; see Methods) were used as continuous predictor variables in a common general linear model. Consequently, the results reported below reflect the specific effects for word frequency and AoA. Two main questions were addressed. First, are early and late learned words represented differently in the brain and, if so, is the neuroanatomical dissociation between early and late acquired words similar or different from that seen for low and high frequency words? Second, if AoA effects are different from word frequency effects, can theoretical explanations of the AoA effect proposed in the behavioral literature (see above) account for our neuroimaging results?

The activation data reported stem from 12 participants who read 136 words and 136 phonologically legal pseudowords while functional images of their brains were taken from 12 axial slices. The participant's task was to judge whether the presented stimuli were legal German words or not (i.e., to perform a lexical decision task). To determine whether or not the reported results for AoA are modality specific, we also report data from a second study in which 14 participants performed an auditory lexical decision task on the identical words and pseudo-words.

\section{Materials and methods}

\section{Participants}

Twelve native-speakers of German ( 7 males; 20 to 31 years) participated in the visual study after giving informed consent. For the auditory experiment, 14 independent righthanded participants (6 males; 19 to 30 years) were scanned that were drawn from the same pool of subjects. All participants were right-handed (Oldfield, 1971), had normal or corrected-to-normal vision, and were without history of neurological or psychiatric disorders.

\section{Experimental procedure}

In the visual experiment, 136 words and 136 pseudowords (of four to seven letters and one to two syllables in length) were projected for $400 \mathrm{~ms}$ onto a translucent screen that participants could view through mirrors mounted onto plastic glasses. The stimulus onset asynchrony (SOA) was $7.5 \mathrm{~s}$. For the auditory study, the same set of words and pseudo-words was spoken by a trained female speaker, digitized and edited, and, during the functional measurements, presented via headphones to the participants. Stimulus onsets were jittered with a mean SOA of $6 \mathrm{~s}$ in this study. Response keys were registered from the onset of the stimuli in both studies. Stimuli were presented in a singletrial design. The stimulus order was determined randomly with the constraints that no more than three items of the same type (i.e., words or pseudo-words) were presented in a row. In addition, the distribution of event types across the time series and transition frequencies between words and pseudo-words were controlled.

\section{Material}

All word stimuli were inanimate German nouns. Thus, there were no confounds due to word class or animacy possible. Words and pseudo-words, as well as low and high frequency words, were matched for length with respect to number of letters and syllables (see Fiebach et al., 2002). Age of acquisition did not substantially correlate with word length $(r=0.15$ for letters and $r=0.16$ for syllables, both NS). Pseudo-words were phonologically legal non-words constructed from the word stimuli by exchanging one or two letters as described previously by Fiebach et al. (2002).

The words used in the experiment were either of low frequency (i.e., zero or one in one million), or fell into a category of high frequency items that was more variable and had a mean of 149.75 in one million. Frequencies were determined by using the CELEX database (Baayen et al., 1995), which is based on a corpus consisting of written and spoken German text. The values of the frequency regressor used for the present analysis represented logarithmic transformations of these frequencies, as given in the CELEX database.

Age of acquisition values used as the second regressor variable were determined by subjective ratings from a separate sample of individuals drawn from the same pool of subjects as those that participated in the fMRI experiments $(n=29)$. The ratings were performed by using a 7-point scale $(1=2$ years or below, $2=3-4$ years, $3=5-6$ years, $4=7-8$ years, $5=9-10$ years, $6=11-12$ years, $7=13$ years or later) as described in the literature (e.g., Gilhooly and Logie, 1980). Also in analogy to previous studies, participants of this questionnaire study were instructed to rate when they subjectively believed they had learned the presented words. It has been demonstrated previously that subjective AoA ratings correlate highly with more objective AoA norms acquired from children through picture naming tasks (Gilhooly and Gilhooly, 1980; Morrison et al., 1997). The mean AoA value across all items was 3.9 (SD 1.4). The distribution of AoA values is displayed in Fig. 1. Subjective ratings of word concreteness were acquired from an independent sample of 30 individuals. Here, a rating scale with four steps from "very concrete" to "very abstract" was used. Word concreteness did not correlate with age of acquisition $(r=-0.04 ; \mathrm{NS})$.

\section{fMRI data acquisition and analysis}

In the visual word processing study, images from 12 axial slices (FOV $19.2 \mathrm{~cm}$; voxel size $3 \times 3 \times 5 \mathrm{~mm}$; interslice distance $2 \mathrm{~mm}$ ) were acquired with a 3-T Bruker Medspec 30/100 scanner using a BOLD-sensitive gradient echo EPI sequence $(\mathrm{TR}=1.5 \mathrm{~s}$; $\mathrm{TE}=30 \mathrm{~ms})$. In the 


\section{A Fiebach et al., $2002 \quad$ B Regression Analysis}
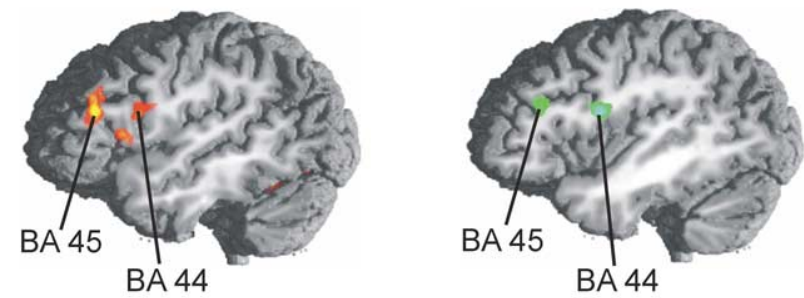

$\mathrm{p}<.0025 \square \mathrm{p}<.0000$

$p<.0025$

$p<.0001$

Fig. 2. Activation results for the word frequency effect in visual word processing. Shown are 3D renderings of brain activity modulations by word frequency. (A) Data from the study by Fiebach et al. (2002) in which high and low frequency words were compared in a direct contrast (i.e., low $>$ high frequency). Increased activity for low frequency words compared to high frequency words was observed in the left inferior frontal gyrus posterior-superiorly in the pars opercularis (BA 44/6;-42, 1, 17; 30 voxels; $Z=3.98$ ) and more anteriorly in the pars triangularis (BA $45 ;-51$, 31 , 10; 31 voxels; $Z=4.17$; reproduced with permission of MIT Press). (B) Brain activation results for the present multiple regression analysis of the visual lexical decision study (coded in green to blue here) replicate the findings from the previous analysis. Activity in pars triangularis (BA 45; $-43,29,7 ; 10$ voxels; $Z=3.07$ ) and pars opercularis (BA 44/6; $-43,3$, $13 ; 17$ voxels; $Z=3.66$ ) of the left inferior frontal gyrus increased with decreasing word frequency (not shown in this figure is activity in the left anterior insula at $-32,14,-4 ; 22$ voxels; $Z=3.66$ ).

auditory experiment, only 10 slices were acquired with a repetition time of TR $=2$ in order to reduce scanner noise and facilitate the understanding of auditorily presented stimuli. The other parameters remained constant. Prior to functional imaging, T1-weighted MDEFT images (data matrix $256 \times 256, \mathrm{TR}=1.3 \mathrm{~s}, \mathrm{TE}=10 \mathrm{~ms}$ ) were obtained with a non-slice-selective inversion pulse followed by a single excitation of each slice (Norris, 2000) for coregistration of functional scans with previously obtained high resolution whole-head 3D MDEFT brain scans (128 sagittal slices, 1.5 -mm thickness, data matrix $256 \times 256$ voxels; see Lee et al., 1995). T1-weighted structural EPI images $(\mathrm{T} 1=1200$ $\mathrm{ms}, \mathrm{TR}=30 \mathrm{~s}, \mathrm{TE}=30 \mathrm{~ms}$ ) were also acquired to allow for a refinement of the coregistration of functional datasets to the high resolution structural scans.

All data analyses were performed with the LIPSIA software package (Lohmann et al., 2001), which implements the statistical routines of SPM. After preprocessing (consisting of motion correction, correction for slice time delays, baseline correction, and spatial smoothing with a Gaussian kernel of $5.6 \mathrm{~mm}$ FWHM) and coregistration into normalized stereotachtic space (Talairach and Tournoux, 1988), functional datasets were analyzed in an event-related design (random effects model) with two event types (i.e., words and pseudo-words) and two continuous regressors to model the effects of AoA and word frequency on brain activity elicited by word stimuli (Friston et al., 1995; Josephs et al., 1997). The regressor for AoA was determined by entering the average AoA ratings from the questionnaire study into a parameter with one value for every word in the appropriate order in which the items were presented. The discrete parametric variable was entered into a general linear model estimation during which it was convolved with a Gaussian kernel. The resulting continuous vector (with one value for every time step) was later used as regressor in the model estimation of the common general linear model. The analogous procedure was performed for logarithmic frequency. The design matrix with the two regressors and the functional data were convolved by using a Gaussian kernel with 4-s FWHM and a high pass filter set to $1 / 80 \mathrm{~Hz}$.

The neural correlates of AoA and word frequency were investigated by calculating contrast images reflecting the effects of either factor given that the respective other factor was kept constant (i.e., when the respective other regressor was set to zero in the contrast vector). Thus, the results reported below reflect only the specific variance component accounted for by either of the two factors, but not the common variance component. Individual contrast images were treated as random effects and submitted to a group statistics (i.e., to a one-sample $t$ test; see Holmes and Friston, 1998) to determine whether the contrasts of interest were reliably different from zero in our sample. Statistical parametric maps (SPM $\{Z\}$ ) were thresholded at $P<$ 0.0025 (uncorrected) at the voxel level. To reduce the probability of accepting false positives, only clusters of significant size ( $P<0.05$, corrected for multiple comparisons), as determined by using the theory of Gaussian random fields (Worsley et al., 1996; Kiebel et al., 2000), are considered.

\section{Results}

\section{Visual lexical decision task}

Whereas both AoA and word frequency reliably predicted visual lexical decision times (AoA: $F=9.75, P<$ 0.002; frequency: $F=44.07, P<0.000$ ), only AoA predicted error rates $(F=9.79, P<0.002)$. This analysis revealed that later learned words and low frequency words were more difficult to process (i.e., elicited longer decision times and more errors). Hence, as predicted both factors reliably affected lexical decision performance.

An analogous regression analysis was performed on the neuroimaging data of the visual lexical decision study. Separate regressors for AoA and frequency were used in a common general linear model during parameter estimation. To identify brain areas specifically associated with AoA and frequency, the specific variance component accounted for by each regressor was assessed while the second factor was partialed out; i.e., contrasts were set such that the effect of one factor was assessed while the other was set to zero and kept constant. The analysis of the frequency regressor yielded only nonsignificant clusters, i.e., clusters with voxels passing the specified significance threshold but which were too small in size to pass the corrected threshold at the cluster size level. As these activations, however, exactly 


\section{visual lexical decision task}

\section{A early > late}

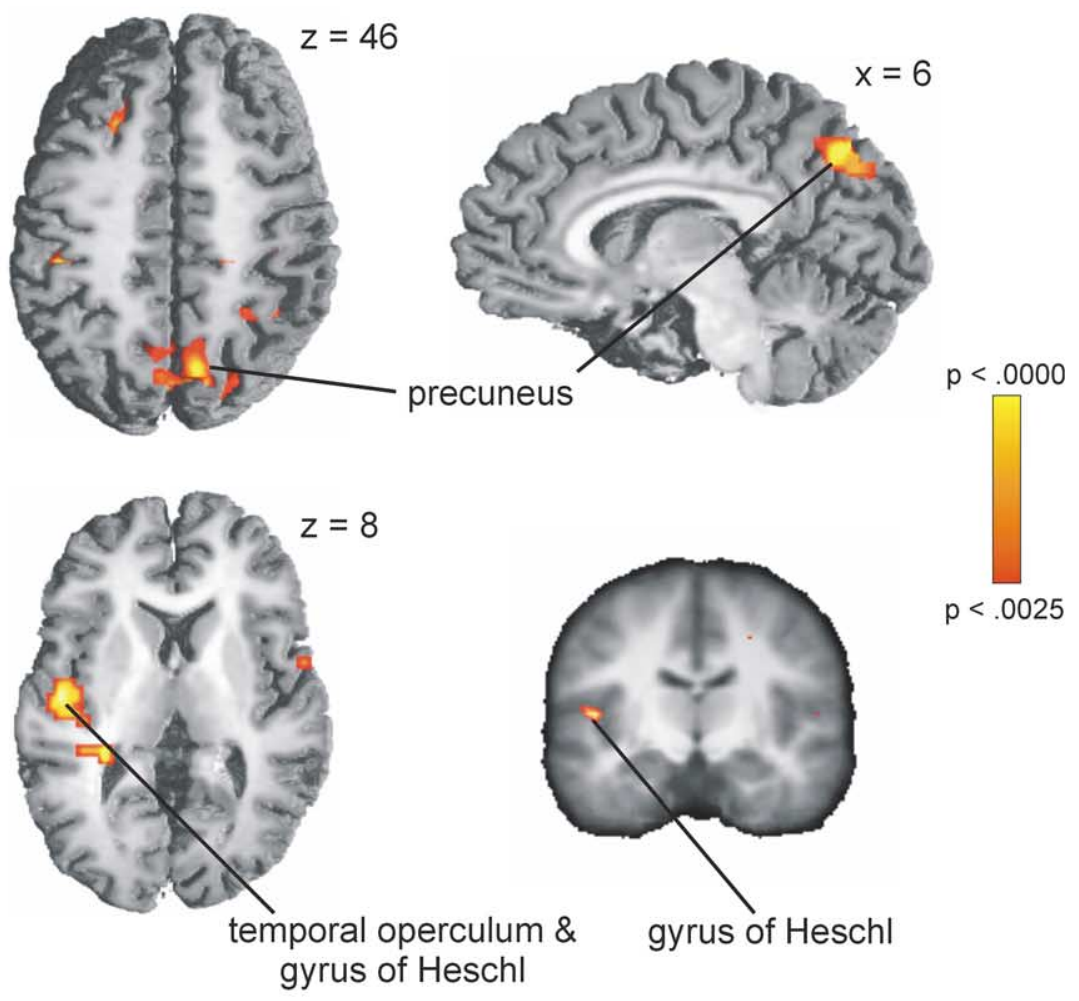

\section{B late > early}
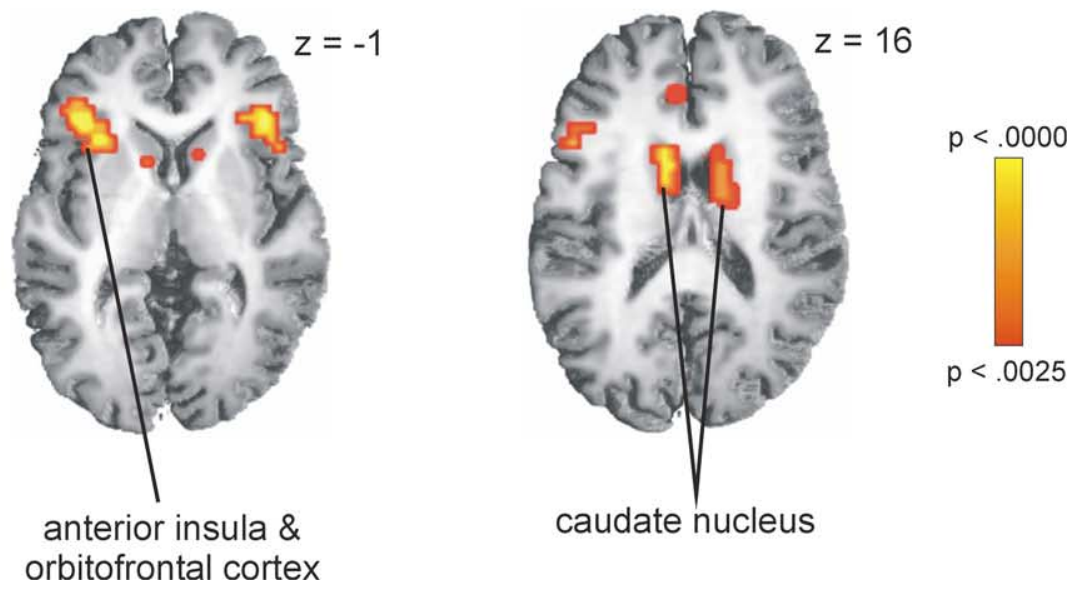

Fig. 3. Activation results for the age of acquisition effect in the visual lexical decision task $(P<0.0025$, uncorrected voxel level, and $P<0.05$, corrected cluster level). Shown are 3D renderings of brain activity changes correlated with word age of acquisition. (A) Increased activity for processing of early learned words was found bilaterally in the precuneus (BA 7b; shown in axial and sagittal sections) and in the left temporal operculum (covering also the gyrus of Heschl/auditory cortex). The lower right panel shows Heschl's gyrus activation overlaid over the averaged anatomy of the 12 participants. (B) Later acquired words elicited increased activity in left inferior frontal and anterior insular regions (BA 45/47/12; left panel) and bilaterally in the caudate nucleus (right panel). Activity observable in the right insula did not pass the cluster size criterium for significance (see Table 1).

replicated the results previously obtained when frequency was analyzed as a discrete variable (i.e., low vs. high frequency, Fiebach et al., 2002; and Fig. 2A, , see also Chee et al., 2002), the voxels passing the significance threshold are visualized in Fig. 2B and briefly reported here nevertheless. In the present regression analysis, low frequency words 
Table 1

Brain activations for the age of acquisition effect in the visual and auditory lexical decision task, as well as in a cross-modal analysis

\begin{tabular}{|c|c|c|c|c|c|c|c|c|}
\hline \multirow[t]{2}{*}{ Brain region } & & \multirow[t]{2}{*}{$\mathrm{BA}^{\mathrm{a}}$} & \multicolumn{3}{|l|}{ Cluster } & \multicolumn{3}{|c|}{ Coordinates } \\
\hline & & & Voxels & $P_{\text {corrected }}$ & $Z_{\max }$ & $X$ & $Y$ & $Z$ \\
\hline \multicolumn{9}{|c|}{ Visual study: early learned words $>$ late learned words } \\
\hline Precuneus & Left/right & $7 \mathrm{~b}$ & 139 & $<0.000$ & 4.65 & 6 & -67 & 39 \\
\hline $\begin{array}{l}\text { Temporal operculum/gyrus } \\
\text { of Heschl }\end{array}$ & Left & 41 & 22 & $<0.1$ & 4.98 & -44 & -10 & 9 \\
\hline \multicolumn{9}{|c|}{ Visual study: late learned words $>$ early learned words } \\
\hline $\begin{array}{l}\text { Inferior frontal gyrus (pars } \\
\text { triangularis and orbitalis) } \\
\text { and anterior insula }\end{array}$ & Left & $45,47 / 12$ & 57 & $<0.001$ & 4.46 & -38 & 34 & 3 \\
\hline Anterior insula ${ }^{\mathrm{b}}$ & Right & & 23 & $<0.1$ & 3.7 & 37 & 24 & -3 \\
\hline \multirow[t]{2}{*}{ Caudate nucleus (head) } & Left & & 38 & $<0.05$ & 3.88 & -11 & 13 & 18 \\
\hline & Right & & 45 & $<0.01$ & 4.78 & 9 & 13 & 13 \\
\hline \multicolumn{9}{|c|}{ Auditory study: early learned words $>$ late learned words } \\
\hline Precuneus ${ }^{\mathrm{c}}$ & Right & $7 b$ & 53 & c & 3.31 & 10 & -55 & 42 \\
\hline \multicolumn{9}{|c|}{ Auditory study: late learned words $>$ early learned words } \\
\hline $\begin{array}{l}\text { Superior temporal gyrus, } \\
\text { anterior portion }\end{array}$ & Left & 22 & 35 & $<0.025$ & 4.51 & -53 & -1 & 0 \\
\hline $\begin{array}{l}\text { Superior temporal gyrus, } \\
\text { anterior-to-middle } \\
\text { portion }\end{array}$ & Right & 22 & 54 & $<0.005$ & 4.02 & 57 & -4 & 6 \\
\hline \multicolumn{9}{|c|}{ Conjunction of visual and auditory data: early learned words $>$ late learned words } \\
\hline Precuneus & Left/right & $7 \mathrm{~b}$ & 63 & $<0.005$ & 4.21 & 10 & -58 & 41 \\
\hline \multicolumn{9}{|c|}{ Conjunction of visual and auditory data: late learned words $>$ early learned words } \\
\hline $\begin{array}{l}\text { Inferior frontal gyrus and } \\
\text { anterior insula }\end{array}$ & Left & 45 & 69 & $<0.001$ & 4.48 & -24 & 23 & 3 \\
\hline
\end{tabular}

a BA, Brodmann's area. Coordinates given are stereotaxic coordinates according to the brain atlas of Talairach and Tournoux (1988). They reflect the location of greatest activity in a given cluster of activation.

${ }^{\mathrm{b}}$ Right hemispheric insula activation for late learned words in the visual study was not significant at the cluster level and was, therefore, not discussed. It is however included in the table as it is also visible in Fig. 3B.

${ }^{\mathrm{c}}$ Activity in the precuneus in the auditory study was tested as an a priori hypothesis based on the visual study. For this test, a lowered statistical threshold of $P<0.05$ (uncorrected) was used.

resulted in stronger activity in the pars triangularis (BA 45) and superior pars opercularis (BA 44) of the inferior frontal gyrus of the left hemisphere (Fig. 2B), as well as in the left anterior insula. The results for word frequency were much weaker in the present analysis than in the analysis described in Fiebach et al. (2002) in which the variance associated with the factor AoA was not partialed out. This suggests that a critical portion of the activity previously attributed to word frequency stems in fact from a variance component shared by frequency and AoA.

When the effect of frequency was held constant, the regression of AoA on the haemodynamic responses yielded reliable activation effects in a set of neural structures distinct from those observed for word frequency (Fig. 3 and Table 1). As Fig. 3A shows, increased activity for early acquired words was observed in the precuneus bilaterally (BA 7b). In addition, increasing activity for early acquired words was detectable in the left temporal operculum, encompassing also the middle portion of the gyrus of Heschl (i.e., of auditory cortex). This latter activation cluster only approached significance at the cluster level $(P<0.1)$. It was, however, the one cluster of activity in the present study with the strongest statistical effect [i.e., $T_{\max }(12)=10.16$, $\left.Z_{\max }=4.98\right]$. It thus appears that the activity observed in the temporo-opercular region is a circumscribed but highly reliable effect.

Processing of later acquired words led to increased activity in the pars triangularis (BA 45) and pars orbitalis (BA 47/12, according to Petrides and Pandya, 1994) of the left inferior frontal gyrus, extending into the anterior insula (Fig. 3B and Table 1). Additional activity for late learned words was observed subcortically in the caudate nuclei of both hemispheres.

\section{Auditory lexical decision task}

To determine whether the observed AoA effects were modality specific or not, data from an auditory lexical decision experiment with the identical stimulus words but an independent set of individuals were analyzed in the same way. Only AoA was a reliable predictor of lexical decision times $(F=16.92 ; P<0.000)$. For the error rates, AoA only approached significance $(F=2.76 ; P<0.1)$, whereas 
frequency was a reliable predictor for error rates $(F=6.92$; $P<0.01)$.

When the fMRI analysis was performed at the statistical threshold used in the analysis of the visual experiment, no specific activity in the temporal operculum or precuneus-or in any other brain area-was observed for early acquired words. Activity in the temporo-opercular region and in the precuneus was subsequently tested at a locally lowered threshold of $Z>1.96$ ( $P<0.05$, uncorrected). This was done because we had the strong a priori expectation of obtaining activation differences in the same brain areas as in the visual study. No effects were found in the temporal operculum. There was, however, a clear increase of activity for earlier learned words in the precuneus with an activation maximum in the immediate vicinity of the precuneus activity observed in the visual study (Fig. 4A and Table 1). Processing of later learned words in the auditory lexical decision task led to a selective activation increase in the anterior portion of the superior temporal gyri bilaterally (extending slightly more posteriorly in the right hemisphere; Fig. 4A).

\section{Cross-modal analysis of age of aqcuisition effects}

To test whether the precuneus activity was indeed modality independent, we combined the individual contrast images from the visual $(n=12)$ and the auditory study ( $n$ $=14)$ into a cross-modal random effects model $(n=26)$. In this analysis, strong and reliable activity for early learned words could indeed be found in the precuneus (Fig. 4B and Table 1). Activity in the temporal operculum was detectable but fell short of statistical significance. For late learned words, the cross-modal analysis showed highly reliable activity in the left inferior frontal cortex and anterior insula (Fig. 4B and Table 1). Thus, it appears that these areas are involved in the processing of visually and auditorily presented late learned words.

\section{Discussion}

The current results revealed a number of new findings that shed light on the neural mechanisms underlying word recognition. First and most important, we demonstrated that word age of acquisition indeed does modulate brain activity, a finding that is reported here for the first time. The second important insight is that AoA modulated activity in brain areas not affected by word frequency. This finding provides evidence against the hypothesis that behavioral effects of word frequency and AoA are due entirely to common underlying mechanisms (as suggested, e.g., by Lewis et al., 2001). Word frequency primarily modulates activity in inferior frontal regions associated with lexical access (Fiebach et al., 2002; Chee et al., 2002). The strong correlation between word frequency and AoA and the fact that the word frequency effect was not significant any more when AoA was partialed out suggests that both factors, to a large degree, modulate activity in the same brain areas. However, it also became clear that age of acquisition has a much more distributed effect than word frequency, also modulating activation in the precuneus, temporal lobe, and in additional inferior frontal regions. Thus, whereas word frequency and AoA effects are partly mediated by the same underlying brain mechanisms, AoA also has a specific contribution to brain activation during word recognition that is not shared by word frequency. These specific effects of AoA will be discussed in the following paragraphs. The third important observation from the present study is that the more distributed AoA effects in the fMRI data parallel results in the behavioral literature that have shown stronger effects for AoA than for word frequency (e.g., Morrison and Ellis, 1995). Analogously, AoA accounted for a larger portion of the variance in brain activation relative to word frequency in the present study.

Before discussing the brain activation results in more detail, it is important to discuss one critical issue in the comparison of word frequency and age of acquisition. Although the present study (as well as the relevant behavioral studies on AoA and word frequency) focuses on the mechanisms of adult language processing, it should be noted that AoA is a measure that captures language exposure across development, whereas word frequency as it is commonly used in psychological studies is derived from adult-directed language. It might, therefore, be true that a separate frequency count for infant-directed speech might yield different frequency counts for the early acquired words, and therefore might also reveal a different relationship between word frequency and AoA. This problem, however, holds for the relevant behavioral studies as well and could only be solved by a specific corpus of age-corrected word frequency norms. Such a database, however, to the best of our knowledge, does not exist.

Late learned words, as well as low frequency words, caused greater activity in the left inferior frontal lobe. Thus, one can conclude that there are similarities in the activation patterns elicited during the processing of the two difficult word conditions-although the exact locations of the activation effects differed between frequency and AoA. Whereas low frequency words activated more dorsal regions of the inferior frontal gyrus, including the superior portion of BA 44 on the border to the inferior frontal and the precentral sulcus, the specific effect for late learned words was found in more anterior and ventral areas, located in BA 45 and, tending toward the lateral orbitofrontal gyrus, in BA $47 / 12$. The increase in inferior frontal activity during difficult word processing is compatible with suggestions found in the neuroimaging literature regarding the role of left inferior prefrontal cortex (LIPFC) in semantic processing. LIPFC is assumed to be critical for the effortful or strategic activation of information from the semantic knowledge system (Fiez, 1997; Thompson-Schill et al., 1997; Gabrieli et al., 1998). The present data suggest that this account has to 


\section{A auditory lexical decision task}

$$
\text { early > late }
$$

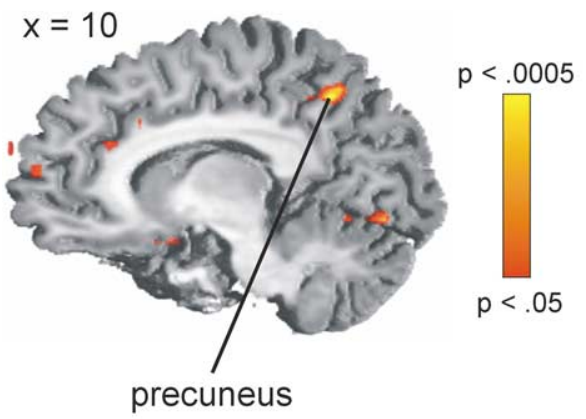

\section{late > early}

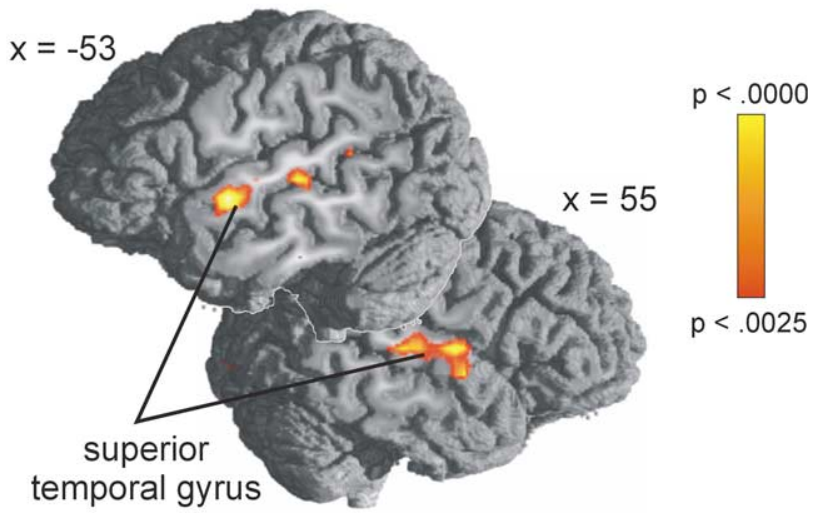

B crossmodal analysis (conjunction of visual and auditory lexical decision task)
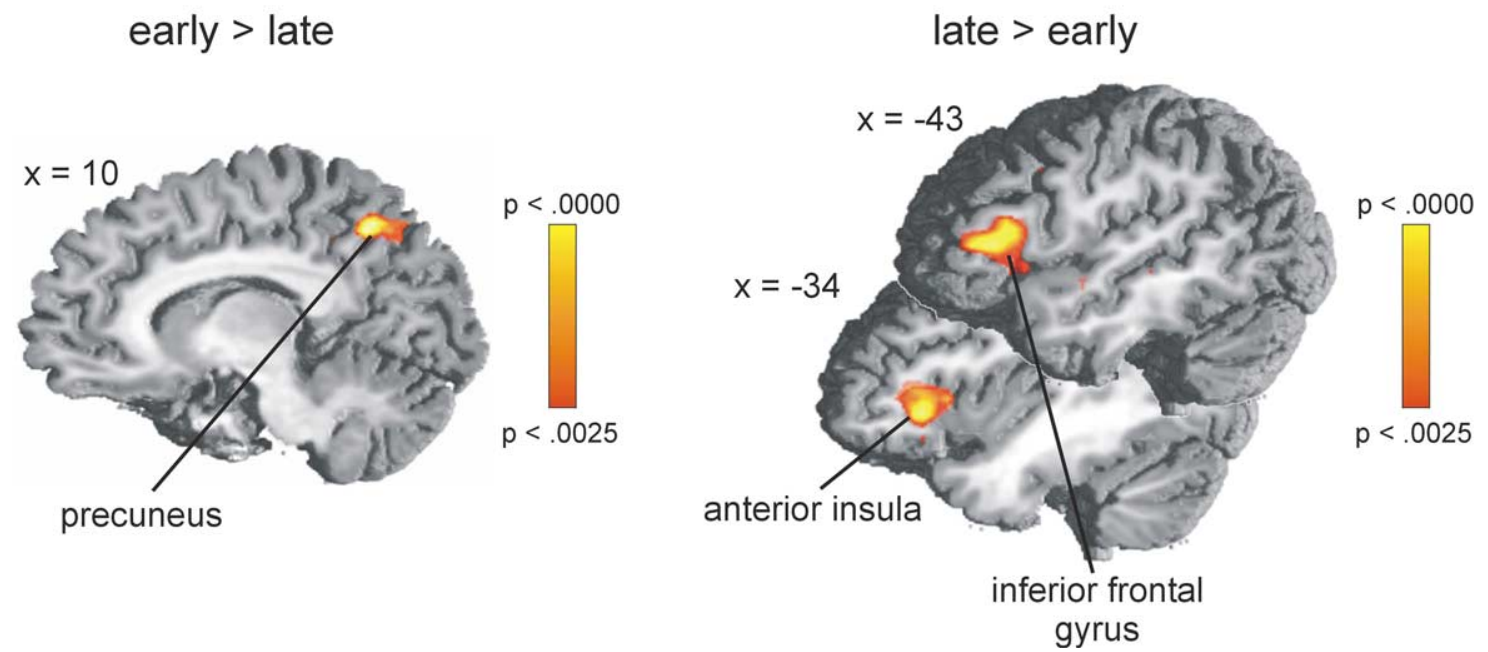

Fig. 4. Activation results for the age of acquisition effect in the auditory lexical decision task and in the cross-modal analysis $(P<0.0025$, uncorrected voxel level, and $P<0.05$, corrected cluster level). (A) Left panel: Precuneus activity for early learned words in the auditory lexical decision task (tested at $P<$ 0.05 , uncorrected). Right panel: Specific activity for late learned words in the auditory experiment was observed in left anterior and right anterior-to-middle portions of the superior temporal gyri (BA 22). (B) In the cross-modal analysis of visual and auditory lexical decision task together, the age of acquisition effect in the precuneus for early learned words was replicated (left panel). Late acquired words elicited specific activity in the left inferior frontal gyrus and anterior insula (right panel).

be extended to the domain of word recognition (as was previously suggested by Fiebach et al., 2002), as it appears that left inferior frontal mechanisms are recruited whenever word recognition cannot proceed smoothly (see Chee et al., 2002, for a similar proposal).

Thus, we propose that whenever word identification is difficult, LIPFC regions provide strategic mechanisms to support the bottom-up processes of word identification. The nature of these strategic influences most likely depends on the exact source of processing difficulty and therefore might determine the specific subregion of LIPFC that is recruited. For the auditory AoA effect, no activity in LIPFC was observed. However, when the conjunction of visual and auditory tasks was calculated, we did observe reliable activity in the same region as was seen in the visual study, indicating that the LIPFC involvement for late learned words is most likely modality independent. This suggests that LIPFC activity for late learned auditory words was probably just below the statistical threshold.

Conversely, facilitation of word processing - as was the case for early learned words - can be caused by different neural mechanisms. Early learned words specifically activated the precuneus and, in the visual lexical decision task, the temporal operculum. For these items, word recognition 
may be facilitated by the fact that additional information is provided by precuneal and primary auditory areas. The precuneus, a medial parietal cortex, has been frequently observed in neuroimaging experiments of episodic memory retrieval (e.g., Shallice et al., 1994; see Rugg and Henson, 2002 , for a recent review). Thus, one can hypothesize, in the case of the AoA effect, that processing early learned words implicitly coactivates contents from episodic memory. It remains a matter of debate, so far, whether activity of the precuneus is specifically associated with the retrieval of imageable memories (as was suggested, e.g., by Shallice et al., 1994) or whether it is involved in episodic memory retrieval in general, independent of imageability (e.g., Krause et al., 1999). As there was no systematic relation between word concreteness (which is very closely related to imageability) and age of acquisition, the present data support the view that the role of the precuneus might be independent of imageability.

Precuneus activation has also been reported for auditory imagery (Yoo et al., 2001) and for the recognition of familiar sounds (Maeder et al., 2001) and voices (Nakamura et al., 2001) - as well as for recognition memory in general (Henson et al., 2000; Herath et al., 2001). Precuneal activity in the present study, thus, might alternatively be taken to suggest that earlier learned words elicit a greater recognition response in the brain. The modality of input did not influence this process, as precuneus activation was detected both in the visual and in the auditory lexical decision tasks. Consequently, recognition responses might be based entirely on information provided through the respective input modality. On the other hand, as indicated by the auditory cortex activation in the visual study, it might also be based exclusively on the acoustic structure of the word that is directly available in auditory word processing but has to be specifically activated in visual word processing. Whatever the exact role of the precuneus in word processing is, its involvement ultimately leads to a facilitation of word processing.

The second area that revealed increased activation for early learned words in the visual lexical decision task was the temporo-opercular region of the left hemisphere. It is especially notable that this activation covered a substantial part of the gyrus of Heschl on which auditory cortex is located. The auditory cortex and surrounding cortical tissue are involved in the processing of acoustic information. Neuroimaging studies have found that auditory cortex in the left hemisphere is specialized for the processing of specific acoustic features of spoken language (Binder et al., 2000; Zatorre et al., 2002). Thus, our results suggest that when reading words learned early in life adults may be activating the sound structure of these words.

It is especially intriguing that temporo-opercular activity for early learned words was observed only in the visual modality. Even when tested at a reduced threshold, this region of the brain did not show an activation increase for early learned words in the auditory study. This result is not unexpected as the auditory cortex, and thus the acousticphonological codes associated with the processed words are automatically activated by the input in the auditory lexical decision task. The modality specificity of the auditory cortex activity for early learned words indicates that there is no need to activate additional acoustic codes for words that are already perceived in this form.

To conclude, the combined results for early learned words from visual and auditory word processing suggest a common role for the precuneus in both modalities, but a special role for acoustic-phonological codes in the visual processing of early acquired words. Words acquired early and later in life do not necessarily differ in length, frequency, or other relevant lexical variables. However, they do differ in the way they are learned. Early in life, i.e., until about 5 or 6 years of age, language is in general learned exclusively through the auditory modality, and understanding the meaning of words is most likely more closely linked to subjective experiences than it is later in life. Accordingly, it is entirely plausible that early acquired words are also represented in the adult brain more strongly in acoustic form and that they evoke a stronger recognition response than later acquired words. The present study provides compelling evidence for the fact that the preponderance of acoustic - and possibly also episodic semantic-information in the mental representations of words learned early in childhood is still observable in adults, and that it facilitates behavior even in a simple word recognition task like the lexical decision task.

This interpretation of our neuroimaging findings for the age of acquisition effect is not completely consistent with the phonological completeness hypothesis (Brown and Watson, 1987), nor is it fully in line with the cumulative frequency account of age of acquisition (Lewis et al., 2001). Although our data indicate that the effects of word frequency and age of acquisition to some degree rely on the same brain areas (as indicated by the nonsignificant frequency effect after AoA was partialed out), we also identified a specific network of cortical brain regions underlying the AoA effect. A third interpretation for the AoA effect reported recently by Ellis and Lambon Ralph (2000) may provide a better framework for the current findings. As already outlined in the Introduction, these authors suggest that due to loss of plasticity during maturation early and late learned words differ in their mental representations formed during cognitive development. Furthermore, Ellis and Lambon Ralph (2000) found that later learned items are harder to "differentiate" than early learned ones. The pattern of brain activity evoked in the present study in adults when they read words acquired at different ages can be taken as support for Ellis and Lambon Ralph's (2000) suggestion of representational differences between early and late learned words. Specifically, they are in line with the notion that later learned words may be more difficult to recognize than earlier ones. However, future studies are needed to investigate this issue more closely. 
The present findings further allow to form more precise hypotheses regarding the nature of the representational difference between these two word types. The finding of specific activity for early learned words in the left auditory areas (for visual word recognition) is compatible with the idea that early learned words are represented in our brain in a more sensory based manner than late acquired words. It is well known that early language experience influences how the brain processes language later in life. It is assumed that neural changes at the level of acoustic or phonetic processing take place very early in life, and lead to a "commitment" to a specific language at the level of the involved neural networks (e.g., Kuhl, 2000). Due to such early changes in the language perception system, it becomes very difficult to overcome the language-specific acoustic-phonetic boundaries later in life when, e.g., learning a second language (e.g., Iverson et al., 2003). On the other hand, it has also been demonstrated that very early (and long forgotten) language experiences can positively influence language processing at adult age (Oh et al., 2003). Although the present data do not directly reflect these early structural changes in language perception, they might very well reflect an aspect of such very early language-learning processes, for example preferred responses to the set of words on which the first experiences with language were made. This hypothesis must be investigated in more detail.

In summary, the current results reveal that age of acquisition plays an important role in word recognition. The study also reveals that AoA modulates activity in areas devoted to semantic retrieval. However, the question remains whether the observed differences are due to a loss of plasticity during maturation, as was suggested on the basis of the neurocomputational simulations by Ellis and Lambon Ralph (2000), or due to differences in the context in which learning took place. It is also worth noting that both the loss of plasticity and context of learning may interact. It is these issues that will continue to spark interest in age of acquisition as an important factor in language processing and its neural representation.

\section{Acknowledgments}

The authors wish to thank Stefan Zysset and Gabriele Lohmann for support in designing the experiments and analyzing the data.

\section{References}

Baayen, R.H., Piepenbrok, R., Gulikers, L., 1995. The CELEX Lexica Database (Release 2) [CD-ROM]. Linguistic Data Consortium, University of Pennsylvania, Philadelphia, PA. See also http://www.kun.nl/ celex.

Binder, J.R., Frost, J.A., Hammeke, T.A., Bellgowan, P.S., Springer, J.A., Kaufman, J.N., Possing, E.T., 2000. Human temporal lobe activation by speech and by nonspeech sounds. Cereb. Cortex 10, 512-528.
Brown, G.D.A., Watson, F.L., 1987. First in, first out: word learning age and spoken word frequency as predictors of word familiarity and word naming latency. Mem. Cogn. 15, 208-216.

Carroll, J.B., White, M.N., 1973. Word frequency and age-of-acquisition as determiners of picture-naming latencies. Q. J. Exp. Psychol. 25, 85-95.

Chee, M.W.L., Hon, N.H.H., Caplan, D., Lee, H.L., Goh, J., 2002. Frequency of concrete words modulates prefrontal activation during semantic judgments. Neuroimage 16, 259-268.

De Moor, W., Ghyselinck, M., Brysbaert, M., 2001. The effects of frequency-of-occurrence and age-of-acquisition in word processing, in: Columbus, F. (Ed.), Advances in Psychology Research, Vol. V. Nova Science Publishers, Huntington, NY, pp. 71-84.

Ellis, A.W., Lambon Ralph, M.A., 2000. Age of acquisition effects in adult lexical processing reflect loss of plasticity in maturing systems: insights from connectionist networks. J. Exp. Psychol. Learn. Mem. Cogn. 26, 1103-1123.

Fiebach, C.J., Friederici, A.D., Müller, K., von Cramon, D.Y., 2002. fMRI evidence for dual routes to the mental lexicon in visual word recognition. J. Cogn. Neurosci. 14, 11-23.

Fiez, J.A., 1997. Phonology, semantics, and the role of the left inferior prefrontal cortex. Hum. Brain Mapp. 5, 79-83.

Fiez, J.A., Balota, D.A., Raichle, M.E., Petersen, S.E., 1999. Effects of lexicaliy, frequency, and spelling-to-sound consistency on the functional anatomy of reading. Neuron 24, 205-218.

Forster, K.I., 1976. Accessing the mental lexicon, in: Wales, R.J., Walkev, E.C.T. (Eds.), New Approaches to Language Mechanisms. NorthHolland, Amsterdam.

Friston, K.J., Holmes, A.P., Worsley, K.J., Poline, J.P., Frith, C.D., Frackowiak, R.S.J., 1995. Statstical parametric maps in functional imaging: a general linear approach. Hum. Brain Mapp. 2, 189-210.

Gabrieli, J.D.E., Poldrack, R.A., Desmond, J.E., 1998. The role of the left prefrontal cortex in language and memory. Proc. Natl. Acad. Sci. USA 95, 906-913.

Gilhooly, K.J., Gilhooly, M.L., 1980. The validity of age-of-acquisition ratings. Br. J. Psychol. 71, 105-110.

Gilhooly, K.J., Logie, R.H., 1980. Age-of-acquisition, imagery, concreteness, familiarity, and and ambiguity measures for 1,944 words. Behav. Res. Methods Instrument. 12, 395-427.

Gilhooly, K.J., Logie, R.H., 1982. Word age-of-acquisition and lexical decision making. Acta Psychol. 50, 21-34.

Grainger, J., 1990. Word frequency and the neighbourhood frequency effects in lexical decision and word naming. J. Mem. Lang. 29, $228-$ 244.

Henson, R.N.A., Rugg, M.D., Shallice, T., Dolan, R.J., 2000. Confidence in recognition memory for words: dissociating right prefrontal roles in episodic retrieval. J. Cogn. Neurosci. 12, 913-923.

Herath, P., Kinomura, S., Roland, P.E., 2001. Visual recognition: evidence for distinctive mechanisms from a PET study. Hum. Brain Mapp. 12, $110-119$.

Holmes, A.P., Friston, K.J., 1998. Generalisability, random effects, and population inferences. Neuroimage 7, S754 [abstract].

Iverson, P., Kuhl, P.K., Akahane-Yamada, R., Diesch, E., Tohkura, Y., Kettermann, A., Siebert, C., 2003. A perceptual interference account of acquisition difficulties for non-native phonemes. Cognition 87, B47B57.

Jescheniak, J.D., Levelt, W.J.M., 1994. Word frequency effects in speech production: retrieval of syntactic information and of phonological form. J. Exp. Psychol. Learn. Mem. Cogn. 20, 687-715.

Josephs, O., Turner, R., Friston, K.J., 1997. Event-related fMRI. Hum. Brain Mapp. 5, 243-248.

Kiebel, S.J., Poline, J.B., Friston, K.J., Holmes, A.P., Worsley, K.J., 2000. Robust smoothness estimation in statistical parametric maps using standardized residuals from the general linear model. Neuroimage 10, $756-766$.

Krause, B.J., Schmidt, D., Mottaghy, F.M., Taylor, J., Halsband, U., Herzog, H, Tellmann, L., Müller-Gartner, H.W., 1999. Episodic re- 
trieval activates the precuneus irrespective of the imagery content of word pair associates. Brain 122, 255-263.

Kuhl, P.K., 2000. A new view of language acquisition. Proc. Natl. Acad. Sci. USA 97, 11850-11857.

Lee, J.-H., Garwood, M., Menon, R., Adriany, G., Andersen, P., Truwit, C.L., Ugurbil, K., 1995. High contrast and fast three-dimensional magnetic resonance imaging at high fields. Magn. Reson. Med. 34 $308-312$.

Lewis, M., Gerhand, B.S., Ellis, H.D., 2001. Re-evaluating age-of-acquisition effects: are they simple cumulative frequency effects. Cognition 78, 189-205.

Lohmann, G., Müller, K., Bosch, V., Mentzel, H., Hessler, S., Chen, L., Zysset, S., von Cramon, D.Y., 2001. LIPSIA-a new software system for the analysis of functional magnetic resonance images of the human brain. Comput. Med. Imaging Graph. 25, 449-457.

Maeder, P.P., Meulli, R.A., Adriani, M., Bellmann, A., Fornari, E., Thiran, J.P., Pittet, A., Clarke, S., 2001. Distinct pathways involved in sound recognition and localization: a human fMRI study. Neuroimage 14, 802-816.

Morrison, C.M., Chappel, T.D., Ellis, A.W., 1997. Age-of-acquisition norms for a large set of object names and their relation to adult estimates and other variables. Q. J. Exp. Psychol. 50A, 528-559.

Morrison, C.M., Ellis, A.W., 1995. Roles of word frequency and age of acquisition in word naming and lexical decision. J. Exp. Psychol. Learn. Mem. Cogn. 21, 116-133.

Nakamura, K., Kawashima, R., Sugiura, M., Kato, T., Nakamura, A., Hatano, K., Nagumo, S., Kubota, K., Fukuda, H., Ito, K., Kojima, S., 2001. Neural substrates for recognition of familiar voices: a PET study. Neuropsychologia 39, 1047-1054.

Norris, D.G., 2000. Reduced power multislice MDEFT imaging. J. Magn. Reson. Imaging 11, 445-451.

Norris, D., Wise, R., 2000. The study of prelexical and lexical processes in comprehension: psycholinguistics and functional neuroimaging, in: Gazzaniga, M.S. (Ed.), The New Cognitive Neurosciences, second ed. MIT Press, Cambridge, MA, pp. 867-880.

Oh, J.S., Jun, S.-A., Knightly, L.M., Au, T.K.-f., 2003. Holding on to childhood language memory. Cognition 86, B53-B64.
Oldfield, R.C., 1971. The assessment analysis of handedness: the Edinburgh Inventory. Neuropsychologia 9, 97-113.

Petersen, S.E., Fox, P.T., Posner, M.I., Mintun, M., Raichle, M.E., 1988. Positron emission tomography studies of the cortical anatomy of single-word processing. Nature 331, 585-589.

Petrides, M., Pandya, D.N., 1994. Comparative architectonic analysis of the human and macaque frontal cortex, in: Boller, F., Grafman, J. (Eds.), Handbook of Neuropsychology, Vol. 9. Elsevier, Amsterdam, pp. $17-58$.

Price, C.J., 2000. The anatomy of language: contributions from functional neuroimaging. J. Anat. 197, 335-359.

Rugg, M.D., Henson, R.N.A., 2002. Episodic memory retrieval: an (eventrelated) functional neuroimaging perspective, in: Parker, A., Wilding, E., Bussey, T. (Eds.), The Cognitive Neuroscience of Memory: Encoding and Retrieval, Psychology Press, London, pp. 3-37.

Shallice, T., Flectcher, P., Frith, C.D., Grasby, P., Frackowiak, R.S., Dolan, R.J., 1994. Brain regions associated with acquisition and retrieval of verbal episodic memory. Nature 368, 633-635.

Talairach, J., Tournoux, P., 1988. Co-Planar Stereotaxic Atlas of the Human Brain. Thieme Medical Publishers, New York.

Thompson-Schill, S.L., D'Esposito, M., Aguirre, G.K., Farah, M.J., 1997. Role of left inferior prefrontal cortex in retrieval of semantic knowledge: a reevaluation. Proc. Natl. Acad. Sci. USA 94, 14792 14797.

Worsley, K.J., Marrett, S., Neelin, P., Vandal, A.C., Friston, K.J., Evans, A.C., 1996. A unified statistical approach for determining significant signals in images of cerebral activation. Hum. Brain Mapp. 4, 58-73.

Yamazaki, M., Ellis, A.W., Morrison, C.M., Lambon Ralph, M.A., 1997. Two age of acquisition effects in the reading of Japanese Kanji. Br. J. Psychol. 88, 407-421.

Yoo, S.S., Lee, C.U., Choi, B.G., 2001. Human brain mapping of auditory imagery: event-related functional MRI study. Neuro report 12, 30453049.

Zatorre, R.J., Belin, P., Penhune, V.B., 2002. Structure and function of auditory cortex: music and speech. Trends Cogn. Sci. 6, 37-46. 